This is the peer reviewed version of the following article: Macchi, M., Wen, J. X., Volkov, K., Heidari, A. and Chung, Y. M. (2015), Modeling liquid fuel cascades with OpenFOAM. Proc. Safety Prog.. doi: 10.1002/prs.11777, which has been published in final form at http://dx.doi.org/10.1002/prs.11777 . This article may be used for non-commercial purposes in accordance with Wiley Terms and Conditions for Self-Archiving. 


\title{
Modelling Liquid Fuel Cascades with OpenFOAM
}

\author{
Marco Macchi ${ }^{2}$, J. Wen ${ }^{1}$, K. Volkov ${ }^{2}$, A. Heidari ${ }^{2}$, Y. Chung ${ }^{1}$ \\ ${ }^{1}$ Warwick FIRE, School of Engineering, University of Warwick \\ ${ }^{2}$ Center for Fire and Explosion Studies, Kingston University \\ *Corresponding author: Jennifer.wen@warwick.ac.uk
}

Keywords: Computational Fluid Dynamics, OpenFOAM, Large Eddy Simulation, Reynolds Averaged Navier-Stokes, Multiphase Flow

\begin{abstract}
Evaporating liquid cascades resulting from gasoline and liquefied natural gas (LNG) tanks overfilling or rupture of elevated pipes create a source of flammable vapour cloud. Such phenomena were responsible for the formation of the large fuel vapour cloud, the ignition of which resulted in the large scale explosion, in Buncefield [1] on 11 December 2005 at the Hertfordshire Oil Storage Terminal, an oil storage facility located by Hemel Hempstead in Hertfordshire, England. Despite its significance, there lacks adequate models treating the underlying physics of this phenomenon. The present study numerically analyses fuel cascades which are considered as a droplet-laden system. Consideration is given to vapour production inside the cascade due to droplets evaporation and breakup. The solver used here is a modification of the sprayFoam solver which is present in the open source computational fluid dynamics (CFD) toolbox OpenFOAM ${ }^{\circledR}[2]$.

The fuel droplets evaporate during their motion and create a cloud of flammable vapour. In order to capture the characteristics of the hazardous phenomena, the CFD model needs to address the underlying physics with adequate sub-models. In the present study, the multi-phase flow is simulated with a combined Eulerian-Lagrangian approach. The governing equations of the gas phase represent the conservation equations of mass, momentum and energy including the source terms arising from the interaction with the droplets. The Reynolds Averaged Navier-Stokes (RANS) simulation approach was used for its computational efficiency. The Large-Eddy Simulation (LES) would be more robust in handling the interaction of the droplets with the flow but it would be require more computational resource. The particulate phase is simulated through a Lagrangian deterministic or stochastic tracking models to provide particle trajectories and particle concentration. Particular emphasis is given to the effect of impingement of droplets to account for the effect of splashing in the impact region. The study involves developing robust and accurate modelling approaches for the instabilities and aerodynamic breakup in the cascade which contribute to the formation of the cloud, air entrainment and fuel impingement on
\end{abstract}


deflector plates. Suitable sub-models have been implemented in OpenFOAM ${ }^{\circledR}$ to facilitate the study.

The predictions are compared with the experimental measurements and CFD predictions previously conducted by Atkinson and Coldrick [3] from the Health and Safety Laboratory (HSL), an agency of the Health and Safety Executive (HSE). The present predictions are found to better capture the interaction between the droplets and the gas phase. Improved agreement with the experimental measurements in the gasoline fuel cascades has also been achieved.

\section{Introduction}

The physics underlying fuel cascades and multiphase flows is complex due to the presence of many factors that have to be modelled. Firstly, the presence of turbulence that is produced by the atmosphere and by the droplet cascade has to be taken into account by the solver using an appropriate turbulence model. Secondly, the solver needs to be able to handle multiphase flow. In the literature, there are currently two main methods that can be used to simulate this type of phenomenon [4], i.e. the Eulerian-Eulerian or the Eulerian-Lagrangian approach. The former considers both the gas and liquid phase as fluids and therefore solves the conservation equations of mass, momentum and energy for both phases while the source terms are modified as a result of phase changes. The second method has been recently used widely in many contexts like fuel sprays, internal combustion engines and other problems related to combustion [5, 6]. Both methods have their limitations. The first approach has more complex formulations but it is computationally more efficient. The second approach has simple formulations but would incur significant increases in computational time. In the present study, the second approach is adopted as it is more often used in this type of flows and also chosen by Atkinson and Coldrick [3]. The droplets are treated as solid particles while the code solves the equation of motion for each particle with the forces acting on it coming from the gas phase. It can also take into account the droplet breakup and heat transfer with the environment.

Another important factor that has been widely discussed in the literature is the splashing model. In the case of a spilling tank or pipe, the fuel will eventually hit the ground and other obstacles. This phenomenon involves complex physics, for which there still lacks adequate sub-models in the literature. Atkinson and Coldrick [3] used a dummy splashing model, as the models available in their chosen CFD code ANSYS CFX ${ }^{\circledR}$ are mostly used for internal combustion engines. The OpenFOAM package offers different models for droplets and wall interactions from the relatively simple sticking, bouncing and escaping conditions to more sophisticated models which consider splashing. It offers a library that can model thin liquid films and splashing of droplet into the film. The splashing model available in conjunction with the film modelling is the Bai and Gosman model [7, 8], which is mostly used for internal combustion engines applications where small droplets hit the walls of the piston. The model involves certain parameters like the critical Weber number and some coefficients which were tuned with experimental data for internal combustion engines. In order for the model to be applicable to fuel cascades applications, these parameters need to be re-tuned with more relevant experimental data. As a first attempt in the lack of such experimental data, both the simple sticking models and the Bai and Gosman model are used in their current status in the present simulations. 


\section{OpenFOAM simulation}

The OpenFOAM CFD toolbox has been used in this study because it is open source and has recently been used many researchers in a variety of problems. In the current study, a new solver CascadeFoam is developed by modifying the existing SprayFoam solver in the OpenFOAM tool box [9].

\subsection{The CascadeFoam solver}

The SprayFoam solver is a transient solver for compressible multiphase flows. It is mostly used for internal combustion engine applications [9]. In order to simulate the fuel cascades, two main modifications have been introduced concerning film modelling and buoyancy treatments.

The second modification is implemented on the momentum and pressure equation (OpenFOAM uses the PIMPLE algorithm, that is similar to the well-known PISO algorithm) in order to consider for buoyancy, which is important in this type of flows, where the density gradients play an important role on the flow field.

\section{$\underline{2.1 .1 \quad \text { Film modelling }}$}

The film modelling library is available in OpenFOAM and is used in other solvers. The library was introduced in Version 2.0.0 and uses a separate mesh to solve the film region; and most of the time this region is simply obtained by extruding one layer from the gas phase mesh. The model was lifted into the new solver, allowing the user to select a splashing model. The assumption of thin film is used. The normal wall velocity is equal to zero and wall-tangential diffusion is considered as negligible compared to wall-normal diffusion. The continuity, momentum and energy equations are then solved for the film region with the proper source terms that take into account the effects of heat transfer, friction of the ground and splashing of droplets on the film. As stated before, the only model currently available in the "splashing" library is the Bai and Gosman model [7, 8] apart from the simple droplet-wall interactions of either absorbing or bouncing.

\section{$\underline{2.1 .2 \quad \text { Buoyancy terms }}$}

Buoyancy is an important factor in multiphase flows, especially when the density of the evaporating component has relatively large difference with that of the gas phase. This is indeed the case for LNG, which is much heavier than air when it first evaporates. The size of the domain is also important in terms of the pressure variations as the density varies with height, but this is much less important than the density variation in the cascade. The original momentum and pressure equations exhibited strange behaviour when the gravity field is not set to zero due to a wrong implementation of the buoyancy terms as the SprayFOAM solver was originally intended for internal flows. For CascadeFOAM, this has been corrected to handle external flows such as the fuel cascade dispersing in the atmosphere.

\subsection{Simulation setup}


The simulation was set up to closely mimic the same configuration used by Coldrick et al. [10], aiming at reproducing the experimental results with accuracy. Very few details were available in their reports [10] concerning the problem geometry but it was possible to extract the main parameters. The computational domain is shown in Figure 1.

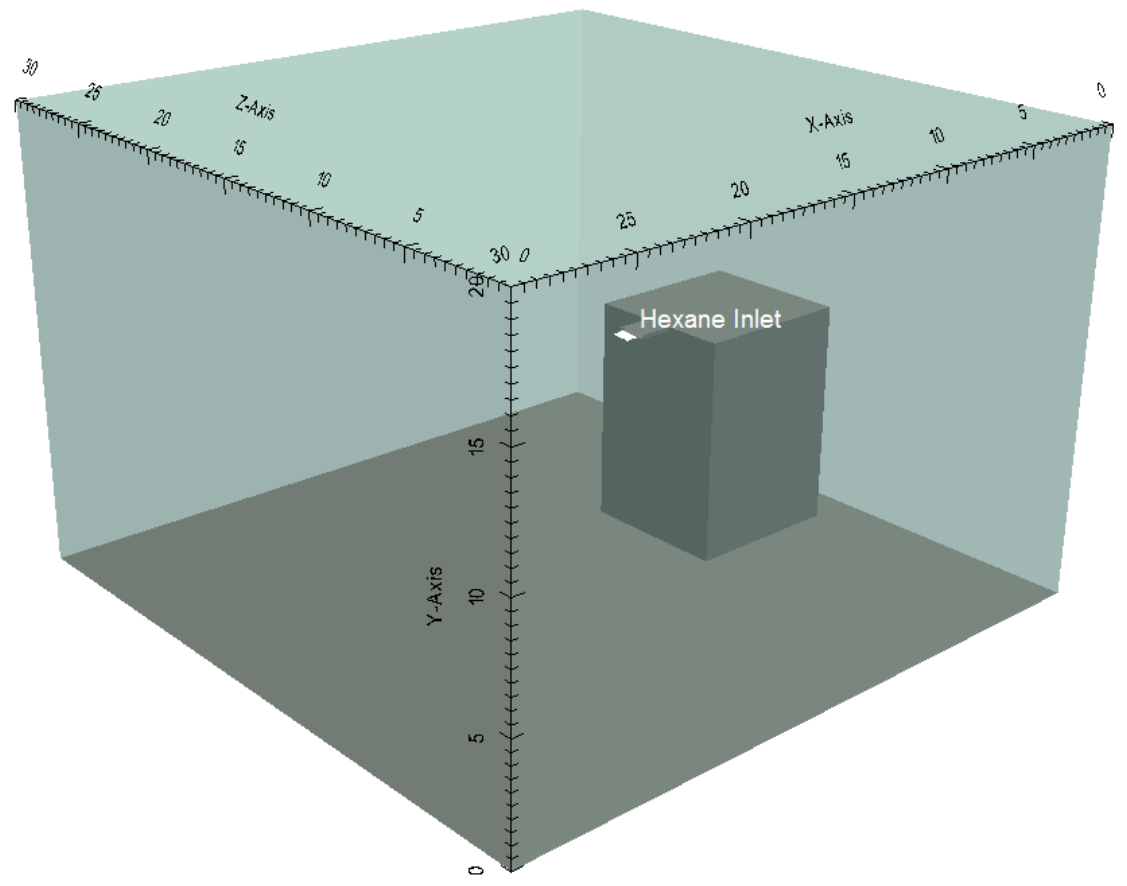

Figure 1: The computational domain.

The dimensions of the domain are about $30 \mathrm{~m}$ for the square base (both $\mathrm{x}$ and $\mathrm{z}$ axis) and $20 \mathrm{~m}$ in height (y axis in the picture). The domain is relatively big in order to take into account the atmospheric movements of the air and the tank is positioned at one side of it. The particles are injected through the region depicted as hexane inlet as depicted in Figure 1. The mass flow rate of hexane at inlet is $11.2 \mathrm{~kg} / \mathrm{s}$. Both the mass flow rate and droplet size distributions are specified using the same values as Coldrick and co-workers [3,10]. The later is based on the RosinRammler distribution with a mean droplet size of $2 \mathrm{~mm}$ and a spreading factor of 3 . The $\mathrm{k}-\omega \mathrm{SST}$ turbulence model [11] is used following Atkinson and Coldrick [3]. Preliminary numerical tests were conducted with the $k-\varepsilon$ model with buoyancy modifications in the transport equations for both $k$ and $\varepsilon$ but the results were less satisfactory than those obtained with the k- $\omega$ SST model. The turbulence intensity was set to $5 \%$ based on a reference velocity of $0.01 \mathrm{~m} / \mathrm{s}$ and a ratio of the turbulent viscosity to the fluid viscosity of 10 following Atkinson and Coldrick [3]. The droplets are considered to be made of hexane with chemical formula $\mathrm{C}_{6} \mathrm{H}_{14}$ and boiling point $68{ }^{\circ} \mathrm{C}$, density $654.80 \mathrm{~kg} / \mathrm{m}^{3}$. Due to heat transfer with the air, the droplets in the cascade start to evaporate as soon as they leave the tank. The latent heat released during the evaporation decreases the droplet and gas temperatures which in turn affect vapour production and the saturation vapour pressure, resulting in the formation of the cold vapour cloud. 
The droplets sub-models were chosen as close as possible to the simulations of Atkinson and Coldrick [3]. In particular, the forces considered acting on the droplets are the aerodynamic drag (which is the sphere drag) and the gravity. OpenFOAM has some more particle forces models but as a first approximation these two were considered to represent the dynamic of the particle parcels properly. The heat transfer between the parcels and the gas phase, which affects the evaporation rate of the liquid hexane is a function of the Nusselt number, which was calculated by the Ranz-Marshall correlation for spheres [13] following Atkinson and Gant [12]. The breakup model used is the widely used Reitz-Diwakar model [14] which considers the breakup process as a function of the Weber number.

\subsubsection{Simulation without splashing}

The first simulation was carried out with no splashing interaction with the ground, in order to verify and better understand the effect of splashing in the vapour production and in the flow field. The parcels of droplets were injected through a rectangular area shown in Figure 2 with a temperature of $6.4^{\circ} \mathrm{C}$ while the surrounding air was at $6^{\circ} \mathrm{C}$. Some factors were not clearly specified in Atkinson and Coldrick [3] such as the maximum and minimum sizes of the droplets, which are needed by the solver in the droplets set up. The trial and error practice was adapted to select the values which would lead to good agreement with the experimental and previous CFD results in Atkinson and Coldrick [3]. The huge number of droplets produced in the cascade is represented by a finite number of parcels within the code, each one consisting of a certain number of droplets with the same size. This is due to the limitation in the computational resources as the computational time is increased by the number of parcels represented. In every time step the gas phase and the droplet phase were solved simultaneously. The two-way coupling approach was used, i.e. the gas phase behaviour affects the droplet motion, thermodynamic quantities and source terms present in the mass, momentum and energy equations; and vice versa.

A close picture of the droplet inlet is shown in figure 2. Clearly, the droplets are scaled with respect to their real size (with a factor of 20) and just a finite number of them are represented because of the limitation in the Lagrangian solver and post-processor. The droplets are colored with their temperature and it can be seen how their temperature decreases while they fall from the top of the tank due to latent heat released from the evaporation process. As stated by Coldrick and co-workers $[3,10]$ the rectangular area was varied during the CFD investigation in order to reproduce the experimental one and understand the effect on the vapour production. In our case the cascade size was fixed to the HSE one to obtain the best agreement with the experimental results. 


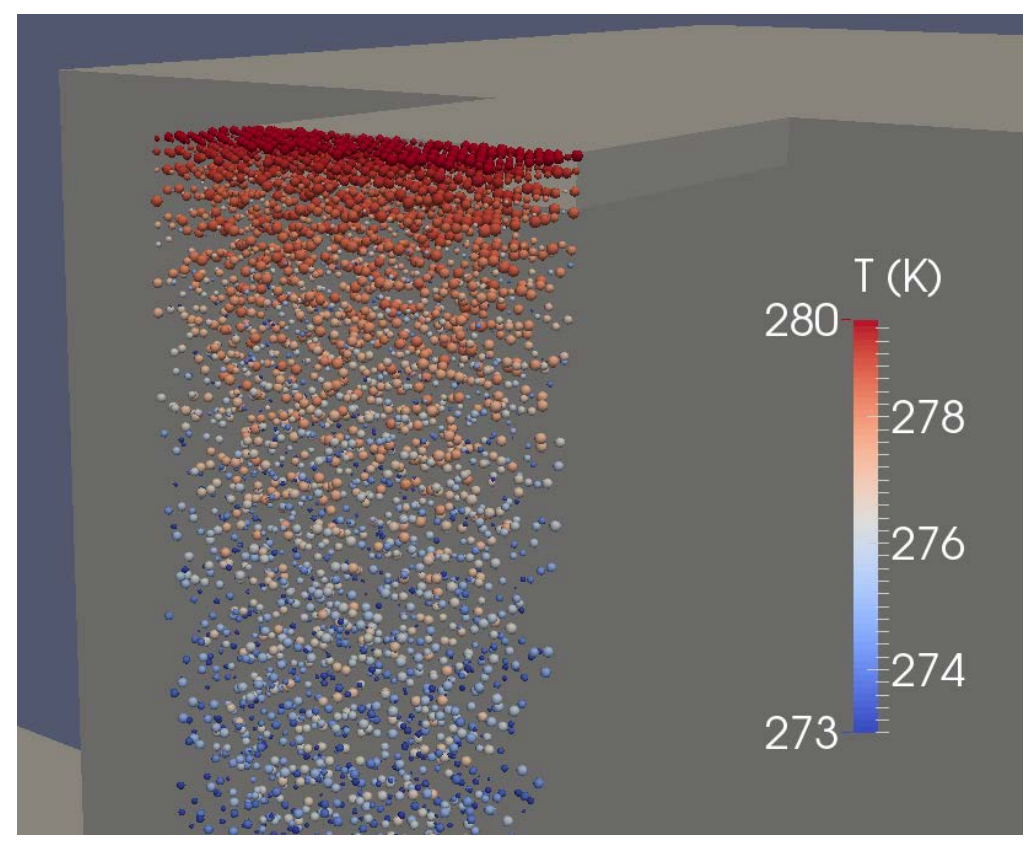

Figure 2: Illustration of the parcels of hexane droplets being injected through a rectangular area.

Figure 3 shows some results from the simulations (the results with the splashing model are also shown, which will be discussed later). The temperature plot for point A shows the lowest value measured in the bulk of the cascade by Coldrick and co-workers $[3,10]$ (both experimental and CFD ones) and in the current simulation. The present results are in reasonably good agreement with their earlier prediction and the experimental measurements. The differences in the transient region are thought to be due to a time delay related to the thermal mass of the aspirated thermocouples and flow recirculation effects within the devices [3].

The steady solution is reached in the CFD simulation after $7 \mathrm{~s}$ from the beginning of the release. For point B which is $0.5 \mathrm{~m}$ above the ground surface and $5 \mathrm{~m}$ from the tank wall, the predictions have captured the overall trend of the measurements but the model over-predicted the measurements. This is thought to be due to the negligence of splashing. In practice, splashing would increase the vapour production and decrease the temperature in the region near the splashing zone [3.10]. The velocity along a line starting from the tank at $0.15 \mathrm{~m}$ height is shown in the last plot. The current predictions are in good agreement with the experimental data $[3,10]$.

\subsubsection{Simulation with the Bai and Gosman splashing model}

The splashing model required more computational time compared to the simulation without splashing. This is mainly because the need to solve the film region which required smaller time steps than the computation of the gas region. The film modelling was represented by a region extruded from the main computational domain. As noted by Atkinson and Coldrick [3], the film modelling only had small contributions to the vapour production in this case, approximately less than $2 \mathrm{~g} / \mathrm{s}$ as measured in the current simulation. For cases where the film is boiling such as LNG 
spilling from tanks, film modelling would play a much more important role as most of the vaporization and dispersion would come from the pool formed. In Figure 5, the predictions are compared with the experimental observations, demonstrating good qualitative agreement in other regions rather than the splashing zone.

The predictions with the splashing model are shown in Figure 3 and compared with the aforementioned results without splashing. The splashing had the effect of decreasing the temperature in the impact region and the vapour production, while the flow field was almost the same as shown in the velocity plot. The main differences in the simulation have been probably caused by the cascade structure and splashing region. The cascade in the CFD simulation was formed by a rectangular injection from the top of the tank while the cascade in the experiments had a parabolic trajectory rather than straight edges as in the CFD simulation. This has probably affected the vapour production and the splashing region. The splashing particles did not eject as far from the splashing region as in the experiments, where they spread out further after hitting the ground and affected the vapor temperature in the far region from the wall. It would hence be possible to improve the predictions by modifying the inlet conditions of the particles and the Bai and Gosman splashing model. As mentioned earlier, several parameters that affect the splashing of particles such as the critical Weber number for splashing, mass ratio of the splashing and absorbed liquid, etc. were pre-determined from experimental data in engine conditions that have much smaller particles than those in the fuel cascades.
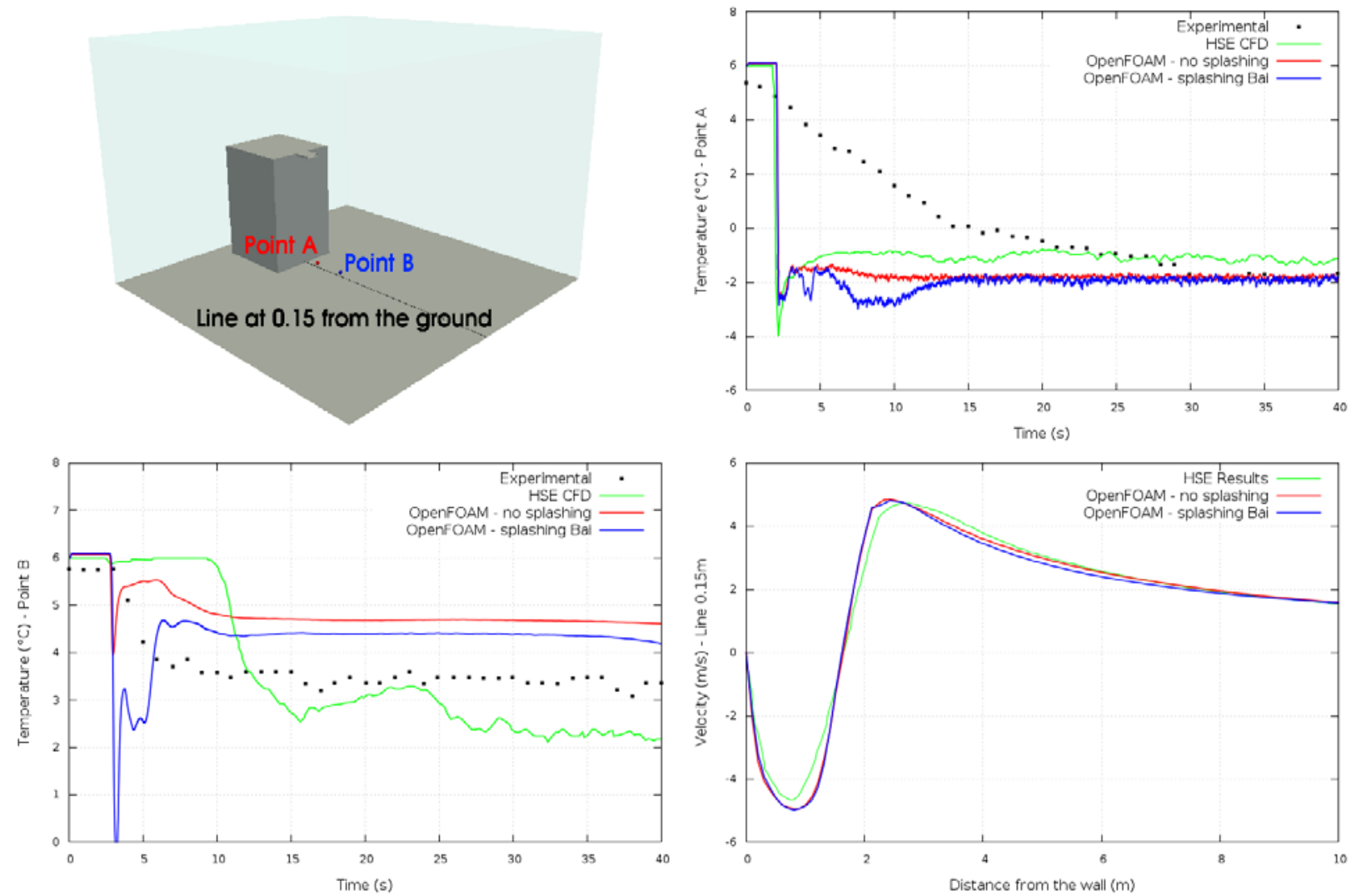

Figure 3: Comparison of the predictions with the numerical and experimental results of Coldrick and co-workers $[3,10]$. 

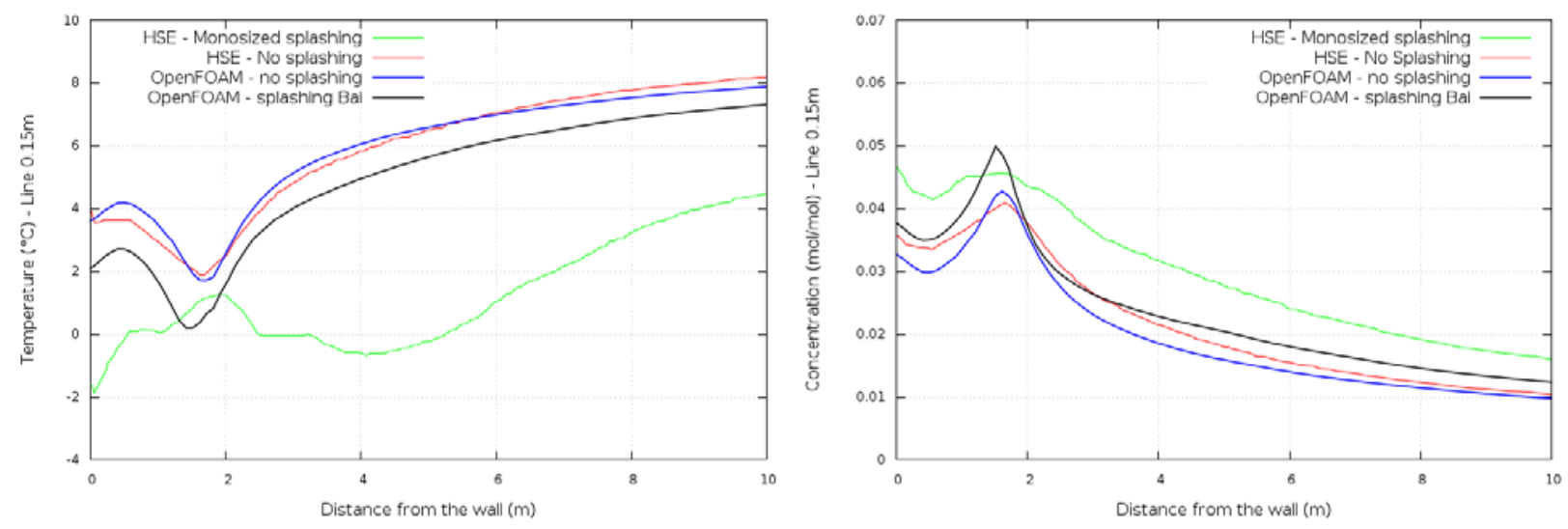

Figure 4: The predicted temperature and fuel concentrations for the same geometric conditions but different initial values of air and fuel temperature.

Figure 4 shows the predictions with the same geometric conditions but different initial values of air and fuel temperature. The initial temperature of the air was set to be $10{ }^{\circ} \mathrm{C}$ in comparison with the hexane at $12.6^{\circ} \mathrm{C}$. The mass flow rate was set to $15 \mathrm{Kg} / \mathrm{s}$. The results without splashing are in good agreement with the predictions of Coldrick et al. [10] in terms of both temperature and concentration, while the predictions with splashing differ more from their predictions. However, in Figure 3, the predictions for point B are somehow in between the current and their predictions. One could deduce that the relatively big differences between the two sets of predicted temperatures in Figure 4 could mean if some experimental measurements were taken, they would probably fall between the two sets of curves.

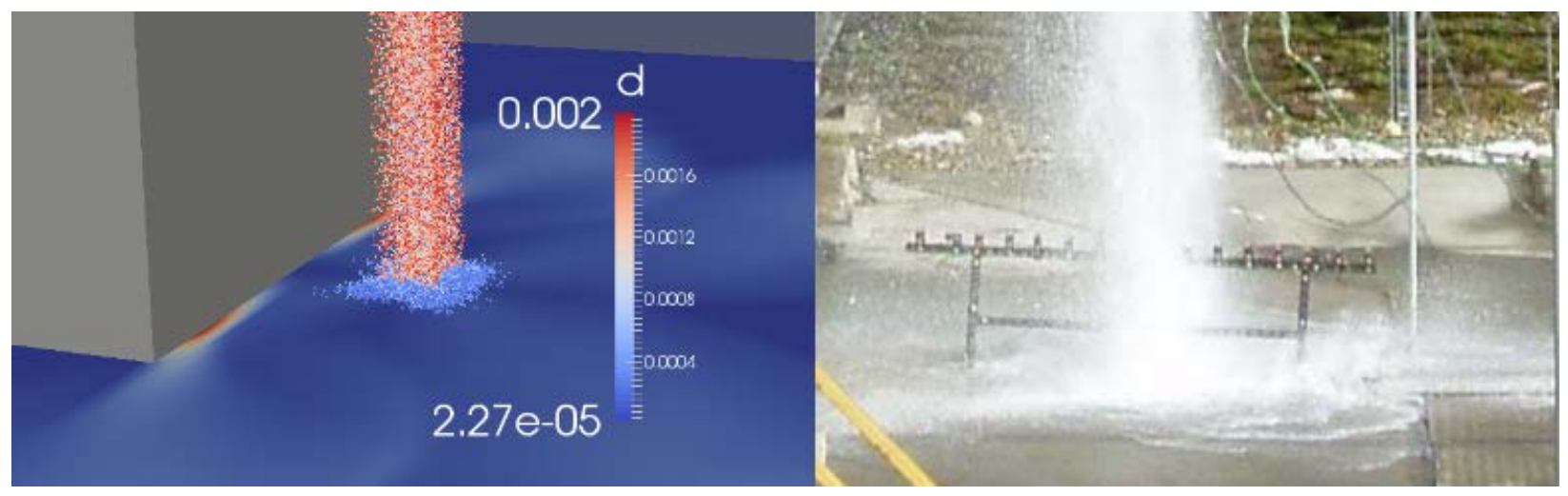

Figure 5: Comparison of the predicted droplet splashing with the experimental observations.

Figures 6 and 7 show the contour plot for the temperature and the turbulent kinetic energy in a plane that cut the CFD domain into half. The temperature is low in the splashing region and the cloud formed by the evaporated hexane in the far region can be seen. A recirculation zone is formed between the splashing zone and the tank wall that can be captured more accurately with the LES approach. 


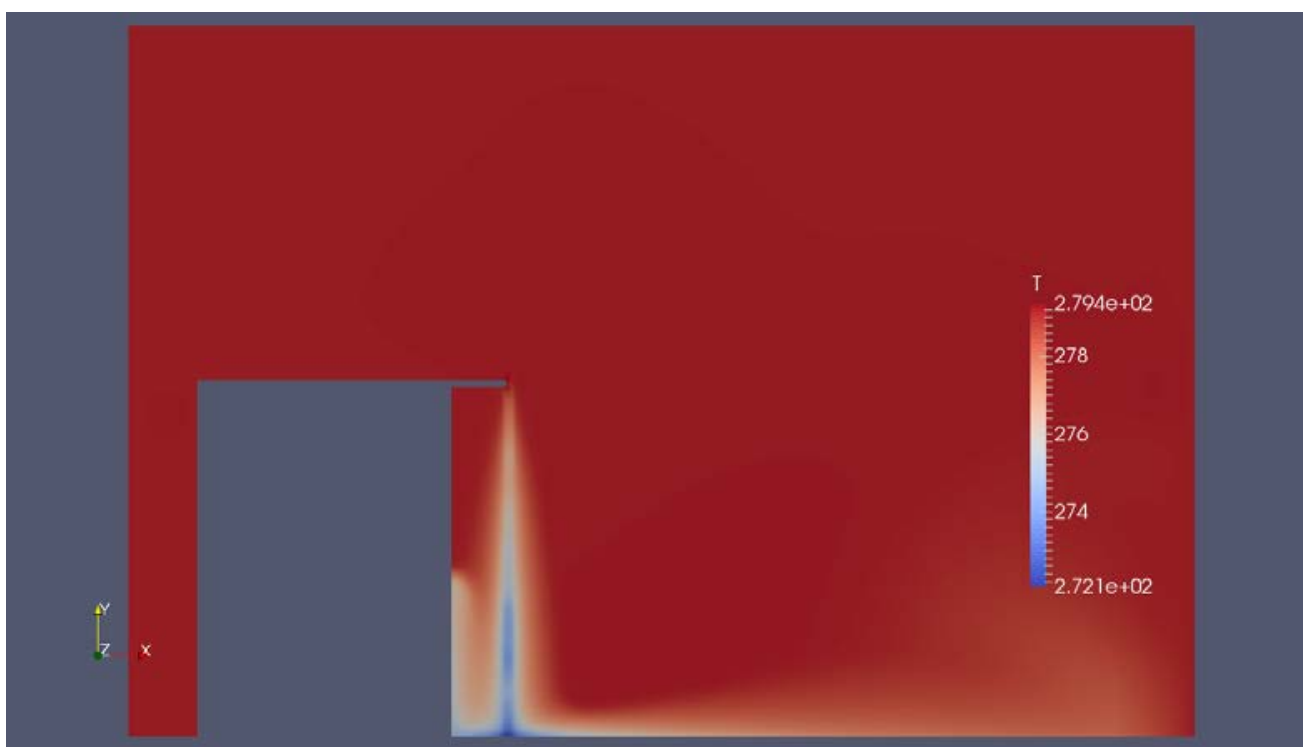

Figure 6: Contour plot of the predicted temperature field at $t=40 \mathrm{~s}$.

Figure 7 shows that the kinetic energy apart from the cascade zone where the Lagrangian phase creates high turbulence compared to the rest of the region, the turbulent kinetic enery in other regions are close to the values set by the boundary conditions. The impact zone has the highest turbulent intensity and therefore the predictions would benefit from local mesh refinement in that region.

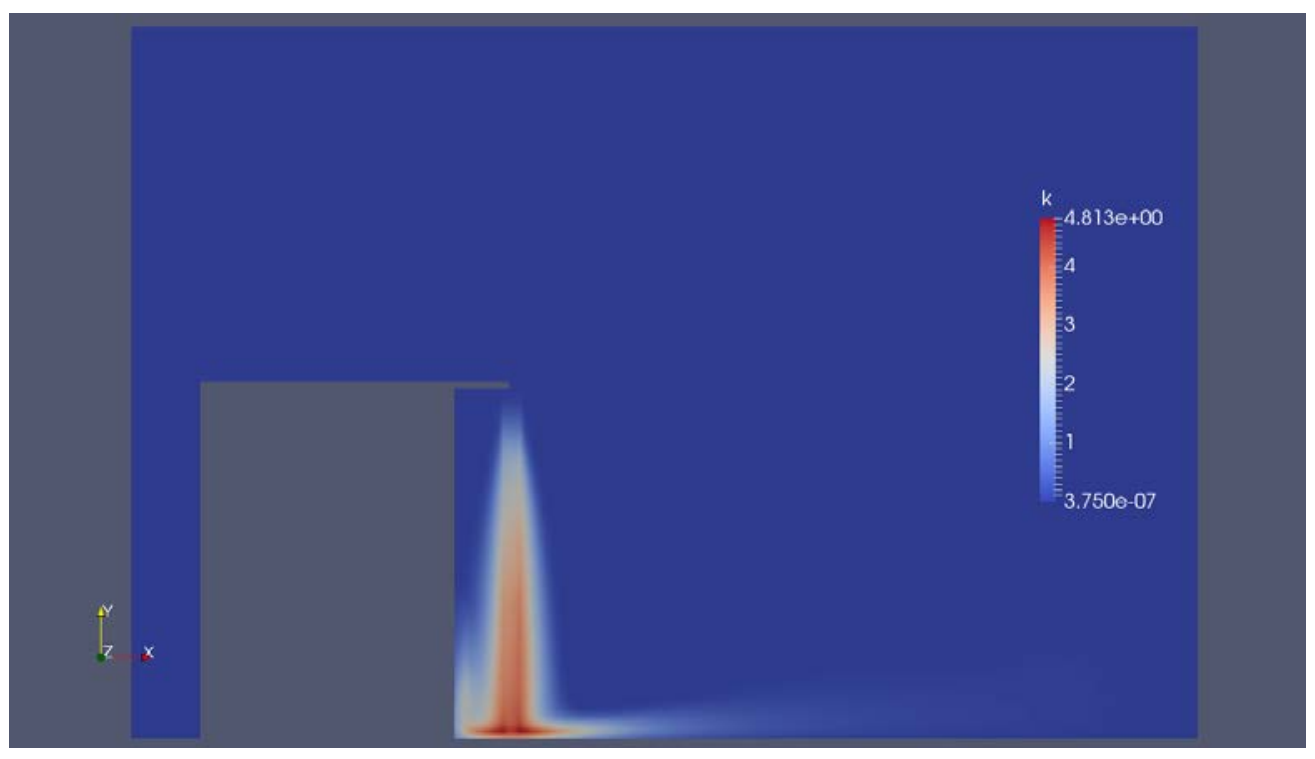

Figure 7: Contour plot of the predicted turbulent kinetic energy at $t=40 \mathrm{~s}$.

Figure 8 shows the 0.5 LFL (Lower Flammability Limit) contours within the computational domain at four different times. It is clear that the cloud develops in the region near the ground. Because of the higher density of hexane in comparison to fresh air, it stays close to the ground. 
The recirculation region between the cascade and the tank wall is also evident resulting in the formation of a cold vapour cloud.

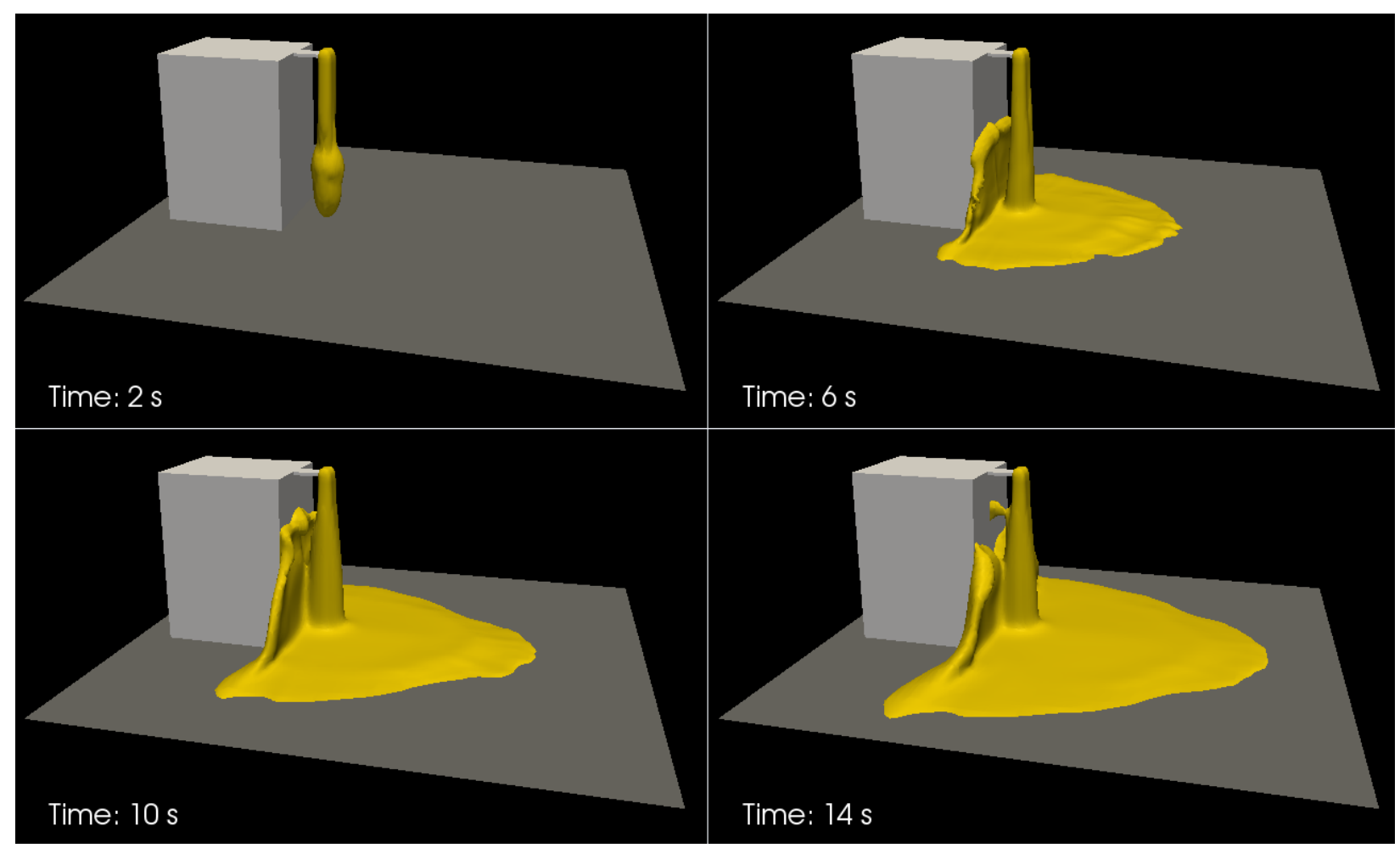

Figure 8: Contour plot of the predicted fuel concentration at 0.5 LFL.

\section{Conclusions}

CascadeFOAM, a new solver for modelling fuel cascades has been developed from SprayFoam within the OpenFOAM toolbox. Both the buoyancy terms in the momentum equations, the film and splashing models have been modified. The predictions have achieved reasonably good agreement with the experimental data and previous predictions of Coldrick et al. [10] for the splashing region. More discrepancy was found in the far field region. This is thought to be due to the chosen splashing model, which contains parameters tuned with experimental data obtained for internal engine conditions. This could be improved by imposing a dummy splashing region following Coldrick et al. [10], but the existing film modelling approach does not permit such approach. Film modelling, as stated above, is very important for cold fuel cascades such as LNG. The results show that the $k-\omega S S T$ model is adequate for the present application. Although the LES approach would better capture the interaction between the droplets and the eddy structures, it is computationally more expensive. The results have also shown that vapour production in fuel cascades is affected by many parameters like the cascade size, droplet size distribution (smaller droplets evaporate more rapidly, while bigger droplets are more likely to splash on the ground) and the splashing at the base of the cascade. The present study has demonstrated the good potential of CascadeFOAM for fuel cascade studies. Further work is underway to improve the splashing model with the view to develop and characterize it for various fuel cascade applications. 


\section{References}

[1] Buncefield Major Incident Investigation Board, Explosion Mechanism Advisory Group Report, 2007.

[2] OpenFOAM 2.3.0, http://www.openfoam.com.

[3] G.T. Atkinson \& S. Coldrick, Vapour cloud formation: Experiments and modelling. Research Report 908, 2012.

[4] C. T. Crowe, Multiphase Flow Handbook. CRC Mechanical Engineering, 2006.

[5] S.E. Gant, A. Heather \& A. Kelsey, CFD Modelling of Evaporating Hydrocarbon Sprays. HSL Report CM/07/04, 2007.

[6] R. Schmehl, G. Maier \& S. Wittig, CFD Analysis of Fuel Atomization, Secondary Droplet Breakup and Spray Dispersion in the Premix Duct of a LPP Combustor. Eighth International Conference on Liquid Atomization and Spray Systems, Pasadena, CA, USA, July 2000.

[7] C.X. Bai, H. Rusche \& A.D. Gosman, Modelling of gasoline spray impingement, 2002.

[8] C.X. Bai, Modelling of spray impingement process. PhD thesis. University of London, 1996.

[9] OpenFOAM, OpenFOAM 2.3.0 User Guide, 2014.

[10] S. Coldrick, S.E. Gant, G.T. Atkinson \& R. Dakin, Factors affecting vapour production in large scape evaporating liquid cascades. Process Safety and Environmental Protection 89, 371-381, 2011.

[11] F. R. Menter, M. Kuntz and R. Langtry, Ten Years of Industrial Experience with the SST Turbulence Model. Turbulence, Heat and Mass Transfer 4, 2003.

[12] G.T. Atkinson \& S.E. Gant, Buncefield Investigation: Liquid flow and vapour production. HSE Research Report 936, 2013. 
[13] W.E. Ranz \& W.R. Marshall, Evaporation from drops, pt. 1. Chemical Engineering Progress, 48, 141-146, 1952.

[14] R. Reitz \& R. Diwakar, Effect of Drop Breakup on Fuel Sprays. SAE Technical Paper 860469, 1986. 